\title{
The influence of nodes sequence and extraneous load induced by graphical overviews on hypertext learning
}

Citation for published version (APA):

Bezdan, E., Kester, L., \& Kirschner, P. A. (2013). The influence of nodes sequence and extraneous load induced by graphical overviews on hypertext learning. Computers in Human Behavior, 29(3), 870-880.

https://doi.org/10.1016/j.chb.2012.12.016

DOI:

10.1016/j.chb.2012.12.016

Document status and date:

Published: 01/05/2013

Document Version:

Early version, also known as pre-print

Document license:

CC BY-NC-ND

Please check the document version of this publication:

- A submitted manuscript is the version of the article upon submission and before peer-review. There can be important differences between the submitted version and the official published version of record. People interested in the research are advised to contact the author for the final version of the publication, or visit the DOI to the publisher's website.

- The final author version and the galley proof are versions of the publication after peer review.

- The final published version features the final layout of the paper including the volume, issue and page numbers.

Link to publication

\section{General rights}

Copyright and moral rights for the publications made accessible in the public portal are retained by the authors and/or other copyright owners and it is a condition of accessing publications that users recognise and abide by the legal requirements associated with these rights.

- Users may download and print one copy of any publication from the public portal for the purpose of private study or research.

- You may not further distribute the material or use it for any profit-making activity or commercial gain

- You may freely distribute the URL identifying the publication in the public portal.

If the publication is distributed under the terms of Article 25fa of the Dutch Copyright Act, indicated by the "Taverne" license above, please follow below link for the End User Agreement:

https://www.ou.nl/taverne-agreement

Take down policy

If you believe that this document breaches copyright please contact us at:

pure-support@ou.nl

providing details and we will investigate your claim.

Downloaded from https://research.ou.nl/ on date: 26 Apr. 2023 
Running head: HYPERTEXT NODE SEQUENCE AND EXTRANEOUS LOAD

\title{
The Influence of Nodes Sequence and Extraneous Load Induced by Graphical Overviews on Hypertext Learning
}

\author{
*Eniko Bezdan ${ }^{\mathrm{a}}$, Liesbeth Kester ${ }^{\mathrm{a}}$, and Paul A. Kirschner ${ }^{\mathrm{a}}$ \\ ${ }^{a}$ Centre for Learning Sciences and Technologies, Open University of the Netherlands \\ P.O. Box 2960, 6401 DL, Heerlen, The Netherlands \\ eniko.bezdan@ou.nl liesbeth.kester@ou.nl paul.kirschner@ou.nl
}

*Correspondence regarding this article should be addressed to Eniko Bezdan, Open Universiteit of the Netherlands, CELSTEC, NL - P.O. Box 2960, 6401 DL, Heerlen, The Netherlands. Tel. +31-45-5762796. Fax.+31-45-5762800. E-mail: eniko.bezdan@ou.nl 


\begin{abstract}
The effects of four hypertext learning environments with a hierarchical graphical overview were studied on the coherence of the node sequence, extraneous load and comprehension. Navigation patterns were influenced by the type of overview provided (i.e., dynamic, static) and whether navigation was restricted (i.e., restricted, non-restricted). It was hypothesised that redundant use of the overview for inducing a high-coherence reading sequence would result in high extraneous load and low comprehension. Coherence was higher in the dynamic than in the static conditions. Coherence was also higher in the restricted than in the non-restricted conditions. Mental effort as a measure of extraneous load was higher at the end than at the beginning of the learning phase, especially in the dynamic restricted and the static nonrestricted conditions, although there was no significant interaction. Comprehension was lowest in the dynamic restricted condition and highest in the dynamic non-restricted and static restricted conditions. Low comprehension in the dynamic restricted condition indicates that overviews can become redundant for reading sequence coherence, negatively impacting comprehension. The evidence suggests that severe restriction of navigation paths should be avoided and that continuous use of overviews such as in dynamic overviews may be detrimental to learning.
\end{abstract}

Keywords: hypertext; graphical overview; coherence; node sequence; extraneous load 


\section{Introduction}

In a hypertext environment, the user must navigate between pieces of linked information (i.e., nodes). Each node contains discrete pieces of information and is linked to one or more other nodes, resulting in a non-linear organisation of content (Conklin, 1987). The way in which information is retrieved from hypertext is influenced by the mental model formed of the interface features of hypertext (Marchionini, \& Shneiderman, 1988). Learning in such environments requires building a mental representation of the structural features of the environment, and at the same time comprehending the content (Schnotz \& Heiß, 2009). Comprehension of text based materials involves building mental representations of the text content which is gradually integrated into the existing knowledge structures of the learner, forming a situation model (Kintsch, 1988; McNamara, Kintsch, Songer, \& Kintsch, 1996). In hypertext environments, building mental representation of the environment's content which meaningfully incorporates the different pieces of information presented at different hypertext nodes are required for successful comprehension (Chalmers, 2003). This process of comprehension, however, is impeded by interruptions in the reading process, which can be a consequence of the elevated navigation requirements associated with the structural characteristics of hypertext. The navigation requirements include having to remember the route to the nodes already visited along with the content displayed on those nodes, and having to decide which nodes to visit next (Müller-Kalthoff, \& Möller, 2006). Salomon and Almog (1998) refer to this as the butterfly defect where learners flutter across information on a computer screen, click on (or do not click on) pieces of information (i.e. hypertext links), to quickly flutter to the next piece of information - not really knowing the value of it, how it relates to other information and without a plan. Learners are seduced into clicking the links, often forgetting what they are looking for. According to Van Merriënboer and Kirschner 
(2012, p. 161), “this fluttering leads - at best - to a very fragile network of knowledge and at worst not even to that. Many a quest ends in a quagmire of charming, but irrelevant pieces of information (and not knowledge)".

Interruptions in hypertext reading occur to the extent the content at subsequently accessed nodes is conceptually unrelated, impeding the development of the situation model (DeStefano \& LeFevre, 2007). Such interruptions represent coherence gaps, as the relationship between the nodes has to be inferred by the reader. A coherence gap which cannot be bridged by inference will lead to a gap in comprehension (McNamara, Kintsch, Songer, \& Kintsch, 1996). However, making inferences is a demanding process as it requires establishing varying types of relationships (e.g., causal, temporal, spatial) between information elements, not explicitly stated in text (Fayol \& Rouet, 2008). Texts low on coherence are known to require high levels of inference which are more likely to be successful when prior knowledge is high (McNamara \& Kintsch, 1996). Similarly, coherence gaps arising from an incoherent reading order of text nodes have been shown to form an obstacle for low prior knowledge, but not for high prior knowledge learners in hypertext comprehension (Salmerón, Cañas, Kintsch, \& Fajardo, 2005).

The cognitive resources required for learning from hypertext are directly related to the structural characteristics of hypertext, as the pathways between nodes (i.e., hyperlinks) determine how information located at different nodes can be accessed (Shapiro \& Niederhouser, 2004). A hyperlink structure which allows for a flexible reading sequence makes it likely that propositions held in working memory will be unrelated to those which are read subsequently (DeStefano \& LeFevre, 2007). Maintaining a coherent mental representation of hypertext content under these circumstances may become increasingly difficult, as the learner must continuously search for corresponding elements in information dispersed at different locations. Search activities as these necessitate undue cognitive 
processing due to split-attention, the tell-tale sign of extraneous load (Chandler \& Sweller, 1991, 1992, 1996). The question which needs to be answered is, therefore: How can 1) the coherence gaps which arise, but cannot be bridged by the learner, and 2) the extraneous load that is caused by search processes be most effectively minimized in order to aid the development of a coherent mental representation of hypertext content?

\subsection{Graphical Overviews and the Reading Sequence of Text Nodes}

The very nature of hypertext which offers a high number of alternative paths, fits intuitively well with the idea of the self-directed learner, actively seeking out information for his or her individual learning needs (Reader \& Hammond, 1994). As an example of technology-enhanced, student-centred learning environments, hypertext is often expected to foster a deeper level of understanding as opposed to more traditional learning approaches (Hannafin \& Land, 1997). Yet, contrary to the high expectations characterising the beginning years of educational hypertext research, empirical evidence indicates that allowing learners to roam freely and without any guidance in hypertext can be detrimental, especially for low prior knowledge learners (Salomon \& Almog, 1998; Shapiro \& Niederhouser, 2004). The high level of user control hypertext affords with its accompanying complexity often cannot be successfully managed by the learner, resulting disorientation (McDonald \& Stevenson, 1996).

One way to limit the complexity of hypertext is to restrict access to its substructures. This was found to reduce feelings of disorientation and improve comprehension for low prior knowledge learners (Müller-Kalthoff, \& Möller, 2006). Also, navigation aids such as graphical overviews of different layout (e.g., linear, hierarchical, network) depicting the structural organisation of nodes are frequently added to hypertext environments. Such navigation aids are assumed to lower the cognitive demands associated with hypertext 
learning by helping with the construction of a mental map of the hypertext environment (Müller-Kalthoff \& Möller, 2003). By aiding navigation, graphical overviews were found to counteract disorientation for low-prior knowledge learners, thus preventing a feeling of being lost (Chen, Fan, \& Macredie, 2006). However, the use of graphical overviews does not seem to be universally advantageous, but seems to be dependent on the prior knowledge of the learner and the structure depicted (Chen, Fan, \& Macredie, 2006; DeStefano \& LeFevre, 2007).

Graphical overviews may not only help navigation by helping to locate individual text nodes, but they also communicate information about the strength of the conceptual relatedness of the content of different text nodes through the structure they depict (Chen, Fan, \& Macredie, 2006). The depiction of conceptual relationships (i.e., lines between concepts) can be used as a guide to identify a coherent reading sequence. The more equivocal this information is, the more difficult it is to identify a coherent reading sequence. In the extreme, every node can be depicted in the overview as having a direct connection to every other node (i.e., every concept pair in the overview is connected with a line), offering no guidance for identifying a coherent reading sequence. The learner might jump back and forth between the graphical overview and/or multiple weakly related text nodes, causing multiple coherence gaps in reading.

There is some evidence suggesting that the structure depicted in graphical overviews has a direct influence on the coherence of the reading sequence of text nodes. If the overview offers unequivocal information of the conceptual relationships which exist between text nodes, a coherent reading order is likely to be followed. It has been observed, that node selection in hierarchical overviews tends to follow a top to bottom and left to right pattern (Amadieu, Van Gog, Paas, Tricot, \& Mariné, 2009), resulting in a more coherent reading sequence in both low and high-prior-knowledge learners than the use of a network overview 
(Amadieu, Tricot, \& Mariné, 2010). In a hierarchy, thematically related topics are presented directly at subordinate and superordinate text nodes or on the same ordination level. In a network (i.e., a heterarchy) ordination levels are less obvious, which means that there is no implied ordering, increasing the likelihood of diminished coherence in reading. Learners' choice of text nodes will be guided by their prior knowledge, with high prior-knowledge learners following a more coherent reading sequence than low prior-knowledge learners (Amadieu et al., 2010).

The increased coherence of the node sequence found with hierarchical overviews may have a positive influence on the comprehension process by activating a coherent proposition network in memory as a representation of text content. According to Kitsch's (1988) construction-integration model, a coherent and consistent proposition network can be easily integrated to fit the context of the text from which it was derived, thereby aiding comprehension. A high-coherence reading sequence should ensure that the propositions derived at subsequent text nodes are highly coherent, reducing the need for search processes which cause extraneous load. Indeed, in hypertext a high-coherence reading sequence was shown to result in lower cognitive load as measured by reaction times to a secondary task, as well as in higher comprehension for low prior knowledge learners, compared to a lowcoherence reading sequence (Madrid \& Cañas, 2009; Puerta Meguizo, Salmerón, Madrid, \& Van Oostendorp, 2009). These studies did not use hierarchical overviews to enhance coherence, nevertheless, they indicate that following a high-coherence node sequence may be an effective way to reduce extraneous load by reducing the need for search processes in hypertext learning.

Hierarchical overviews were associated in a number of studies with better learning outcomes for low prior knowledge learners than network/semantic overviews (Amadieu et al., 2010; Amadieu et al., 2009; Patel, Drury, \& Shalin, 1998; Potelle \& Rouet, 2003; Shin, 
Schallert, \& Savenye, 1994). However, the inclusion of quantitative measures of the coherence of the reading sequence is a relatively recent development in hypertext research examining the influence of graphical overviews. In earlier studies, the reported learning outcomes were explained in terms of variables such as the effects of navigational tools that model expert knowledge to a greater or lesser degree (Patel et al., 1998), the effects of different types of content representation (Potelle \& Rouet, 2003), and the effects of learner control of instruction and advisement (Shin et al, 1994). It is thus still largely unexplored, to which extent the high coherence of the node sequence induced by hierarchical overviews can account for the general finding that these overviews have a positive effect on learning outcomes.

\subsection{Graphical Overviews as a Source of Extraneous Load}

In addition to their differences in the structural depiction of hypertext content, graphical overviews can also differ in the way they enable or disable access to the text nodes. With dynamic/interactive overviews, text nodes can usually only be accessed via the hyperlinks imbedded within the overview; after a hyperlink is selected and clicked, one is brought to the related node, from which a hyperlink leads back to the overview (i.e., backbutton). The lines between the hyperlinks in a dynamic overview are, thus, representations of conceptual relatedness between text nodes, but do not necessarily indicate pathways (i.e., hyperlinks) between them (e.g., Amadieu et al., 2009; Potelle \& Rouet, 2003). This means that between every two text nodes, the dynamic overview has to be opened in order to progress trough the hypertext document. Such restrictions do not apply for static overviews, as they do not contain hyperlinks to text nodes. Navigation proceeds directly from text node to text node and the overview is consulted at the discretion of the learner. When static 
overviews are utilised for visualising possible pathways between text nodes, the lines between the concepts in the overview will correspond to the hyperlinks connecting text nodes, that is, the hypertext structure (e.g., McDonnald \& Stevenson, 1998).

Whether dynamic or static, the schematic representation in graphical overviews is presented separately from the related content at text nodes, as the overview has to be closed before a text node is opened (Chalmers, 2003). Such use of both dynamic and static overviews can be expected to generate extraneous load according to the spatial and temporal contiguity principles of multimedia learning, both specific manifestations of the split-attention effect (Mayer, 2005). However, a situation can be envisaged when the overview is neither a reflection of hypertext structure, nor allows direct excess via embedded hyperlinks to text nodes. Such a static overview would function exclusively as a schematic overview of the content, and no longer as a navigation device which could guide the learner toward a coherent reading sequence. The resulting low coherence reading sequence would become another source of extraneous load (Madrid \& Cañas, 2009; Puerta Meguizo et al., 2009), in addition to the extraneous load induced by the separate presentation of the overview and text nodes.

When a coherent reading sequence of text nodes is likely to be followed without the help of the graphical overview, it is redundant use of the overview which can be expected to cause extraneous cognitive load (for the Redundancy Effect see Kalyuga, Ayres, Chandler, \& Sweller, 2003; Kalyuga, Chandler, \& Sweller, 2000). This might occur when prior-knowledge - either partial or complete - already exists about the relationships between the text nodes or a sub-set of text nodes. Redundant use might be especially prevalent in dynamic overviews, if they are constructed such that they have to be opened after every text node. Some indication suggesting that such continuous use of overviews in hypertext may not be advantageous for learning comes from an eye-tracking study of Salmerón, Baccino, Cañas, Madrid, and Fajardo (2009), who found that reading an overview aids comprehension of difficult texts at the 
beginning of the learning process, but not at the end. Continuous, and therefore, potentially redundant use of static overviews is less likely to occur, since static overviews allow for text node to next node navigation without having to continuously consult the overview.

\subsection{The Present Study}

In order to investigate the combined influence of coherence of the node sequence and extraneous load related to the use of graphical overviews, four conditions were compared for their effect on hypertext comprehension, self-reported mental effort during the learning phase and navigation patterns. The graphical overviews used in all conditions displayed an identical hierarchical structure, as it has been shown in previous studies that a hierarchical structure in overviews promotes a coherent reading sequence of text nodes. The hierarchical structure reflected an expert model of the learning content. To manipulate the frequency with which the graphical overviews are opened, two static overviews and two dynamic overviews were used. The dynamic overviews contained imbedded hyperlinks to text nodes, whereas the static overviews did not. In addition, in one static and one dynamic condition, navigation was restricted to the child and parent nodes ( i.e., nodes that are one step up and/or down in the hierarchy) of the last visited text node, ensuring a high coherent reading sequence of text nodes.

First, node sequence coherence is expected to be higher in the dynamic conditions due to more frequent exposure to the overview than in the static conditions. The highest coherence is expected for the dynamic non-restricted overview, as here learners were expected to adhere to top to bottom and left to right navigation patterns, in line with previous research. The dynamic restricted and static restricted overviews are expected to lead to comparable levels of coherence as both impose the same level of restriction. However, the restrictions in both 
conditions should insure that attention is drawn to only a sub-sets of the hierarchical structure at any one time instead of the entire hierarchy, resulting in lower level of coherence than with the dynamic non-restricted overview. In the static non-restricted condition, the hierarchical structure in the overview is not a reflection of the hyperlink structure of the hypertext environment (i.e., hyperlinks between text nodes) as in the static restricted condition, nor does it allow for direct navigation from the overview to text nodes as in dynamic conditions. Therefore, the static non-restricted overview is hypothesised to have a limited influence on navigation patterns, leading to the lowest coherence.

Second, it is hypothesised that a similar degree of the coherence of the node sequence need not be associated with similar levels of comprehension when the degree of redundant use differs. Thus, redundant use of a graphical overview is predicted to cause high extraneous load and low comprehension, even when coherence is high. As a measure of extraneous load, self-reported mental effort scores were collected during the learning phase. Mental effort during the learning phase is thought to reflect extraneous load related to instructional design (Van Gog and Paas, 2008). In addition to high mental effort and low comprehension, highfrequency use of the graphical overview is also regarded to be a possible indicator of redundant use. The overview in the static restricted condition is a representation of the hyperlink structure between text nodes, and is only expected to be consulted to aid navigation between text nodes when necessary. As a consequence, the lowest redundant use is hypothesised to occur with the static restricted overview, as the overview is only expected to be used intermittently. Further, it is hypothesised that if the obligatory use of dynamic overviews at some point in the learning process becomes redundant, then the even more specific guidance by restricting navigation to only a subset of active hyperlinks of the dynamic restricted overview would represent an even greater redundancy. The highest mental effort and lowest comprehension scores are hypothesised to occur in the static non-restricted 
condition. Even though frequency of overview use is expected to be low, as it is not relevant for navigation, from the point of the coherence of the reading sequence all use could be regarded as redundant. Here, low coherence is expected to be the main source of extraneous load instead of redundancy, and as coherence is expected to be lowest, extraneous load is hypothesised to be the highest in this condition. The summary of the expected effects is given in Table 1 .

$$
\text { *** Insert Table } 1 \text { about here*** }
$$

\section{Method}

\subsection{Participants}

The participants in this experiment were $733^{\text {rd }}$-year undergraduate pedagogy students (mean age $=21.22, \mathrm{SD}=1.92 ; 9$ males and 64 females) at Ghent University, Belgium . Participation was voluntary and was rewarded with a $€ 10$ gift certificate.

\subsection{Materials}

\subsubsection{Learning task.}

Students were required to learn as much as possible about project management by studying an introductory course on this subject presented to them in a hypertext format. Students had to work individually and were not allowed to take notes.

\subsubsection{Hypertext learning environments.}


In this experiment four hypertext learning environments were used in four experimental conditions, namely a hypertext environment with 1) a dynamic (i.e., clickable) graphical overview with navigation restriction, 2) a dynamic graphical overview with no navigation restriction, 3) a static (i.e., non-clickable) overview with navigation restriction, and 4) a static overview with no navigation restriction. The overviews displayed identical hierarchical structures reflecting an expert model of the domain organisation (Figure 1). The 15 concepts in the graphical overviews denoted the 15 text nodes of the hypertext environments. The content on text nodes was presented as a title and a text field and were identical across conditions, and encompassed approximately 3300 words. Some text fields contained only text, while others included text and diagrammatic illustrations of the text content. Content higher in the hierarchy discussed more general topics, whereas content lower in the hierarchy discussed more specific and complex topics related to the content of nodes higher in the same branch of the hierarchic tree. An example of a high level node containing a more general topic is the node describing 'Planning procedure' where the concepts duration, start end completion of activities and delays in activities impacting a project are introduced, but not further explained, and the concept of slack for example is not used at all. A low level node containing a more specific topic is the node 'Slack (CPM)', where the specific technique of calculating the slack by employing the CPM algorithm is explained. Although still related, the content of these two nodes is conceptually distant, as understanding how exactly the content of the node 'Slack (CPM)' is related to the content of 'Planning procedure' is difficult without first reading the intermediate text nodes explaining what a relationship diagram is, what the critical path of a project is, why determining the critical path is essential for the planning of the project, how CPM algorithm is employed to identify the critical path etc. 
In both dynamic conditions, navigation to text nodes was only possible from the graphical overview as text nodes were not connected directly by hyperlinks to each other. The clickable concepts in the overviews represented active hyperlinks to the corresponding text nodes. A hyperlink was presented which led back to the graphical overview (i.e., back-button) in the upper right corner of the screen of each text node. In the dynamic restricted condition, navigation was continuously restricted to the nearest neighbours (i.e., child and parent nodes) of the last visited text node. This was achieved by automated activation and deactivation of hyperlinks in the overview in response to navigation behaviour. Active hyperlinks were denoted in the overview by having a thicker frame. In the dynamic non-restricted condition navigation was unrestricted, which meant that all the hyperlinks in the overview could be clicked at any time. The frame around all the hyperlinks was therefore of identical thickness.

In the static conditions, the overview did not contain imbedded hyperlinks to text nodes. The concepts in the overview here also denoted text nodes, but could not be clicked. In the static conditions, the text nodes contained hyperlinks to other text nodes. The hyperlinks on text nodes were not imbedded within the text content of the nodes, but were displayed separately at the bottom of the screen (see Figure 2). In the static restricted condition, the overview represented not only the expert model of the domain organisation but also mirrored the exact hyperlink structure of the document; a line between two concepts in the overview was a representation of a hyperlink between the two corresponding text nodes. For example, in the graphical overview 'Planning procedure' was connected by lines to 'Project initiation , 'Data gathering' and 'Data analysis (see Figure 1), which meant that the text node 'Planning procedure' had three hyperlinks to these three text nodes (Figure 2). A text node in this condition, thus, contained hyperlinks leading to just its child and parent nodes. In the static non-restricted condition, the overview only represented the expert model of the domain 
organisation. It did not represent the hyperlink structure of the document because each text node had hyperlinks leading to all the other text nodes so that text node to text node navigation was unrestricted (Figure 3). In both static conditions, a hyperlink was presented in the upper right corner of the screen on every text node which opened the graphical overview. Clicking at the 'Close' button at the bottom the graphical overview window (not shown in Figures 1) returned the participant to the last visited node. There was no restriction on the number of times the graphic overview could be opened in both static conditions.

$* * *$ Insert Figure 2 about here $* * *$

*** Insert Figure 3 about here ***

\subsubsection{Logging data.}

Browsing behaviour with respect to time spent studying the hypertext document (i.e., duration of the learning phase), number of times individual text nodes were opened, number of text nodes opened, number of times the graphical overview was opened and the sequence in which nodes were opened was logged in all hypertext environments.

\subsubsection{Mental effort.}

Mental effort as measure of cognitive load was assessed on a 9-point Likert scale (Paas, 1992), ranging from a very little effort to very much effort was measured during the learning phase and the post-test. The learning phase asked: 'How much effort did it cost to you to study the learning material?' while the post-test phase the question was: 'How much effort did it cost to you to answer this question?' 


\subsubsection{Tests.}

Comprehension was measured by two (i.e., test 1 and test 2), 10-question multiplechoice tests, which were equivalent in terms of content and difficulty (Guttman split-half coefficient $=.59$.) As the content on text nodes lower in the hierarchy was a further elaboration of related content presented higher in the hierarchy, test items were constructed to reflect this type of content organisation among text nodes. Therefore, identifying the right answer for a question relating to a particular text node required relating the information presented on this text node with the information presented on one or more text nodes higher in the hierarchy. For example:

The CPM-algorithm is a method for:

a) identifying the critical path and minimal allowed slack for each activity.

b) identifying the different activities of a project.

c) arranging activities along a time axes.

d) identifying the critical path and the maximal allowed slack for each activity.

To identify alternative d) as the correct answer, the participant needed to have an understanding of the concepts critical path, slack, and activities which were discussed on several text nodes in differing contexts.

\subsection{Procedure}

Participants logged into the experiment with a unique username and password, and were then randomly assigned to one of the four hypertext conditions. One of the two tests was given to each participant as prior-knowledge test. Before entering the learning phase, a short tutorial about the navigational properties of the respective hypertext was presented on screen. After reading the tutorial, the learning phase began when participants clicked through to the 
hypertext environment in which the first screen they encountered was the graphical overview. During the learning phase the question about mental effort was asked every 5 minutes. This question about mental effort with the accompanying rating scale was presented in a separate window covering the screen. This window closed automatically when a rating was filled in, after which the participant was returned to the last visited node. The learning phase lasted a maximum of one hour. Participants were allowed to end the learning phase (i.e., studying the hypertext) before the hour was full, but they were advised to do so only when they were confident that they mastered the material. The experiment was concluded with the post-test. Participants in the post-test phase were given both tests. The choice to administer both tests as post-test was intended to keep possible carryover effects from prior-knowledge test to posttest the same for all participants. Participants were asked to rate their mental effort for answering each item of multiple-choice test separately.

\subsection{Design, data preparation and data analysis}

Separate 2X2 ANOVAs were run with the between subjects variables Type (dynamic, static) and Restriction (non-restricted, restricted) for pre-test comprehension scores, outcome measures related to navigation and comprehension scores and mental effort exerted during the test phase. Mental effort scores collected during the learning phase were analysed using repeated measures ANOVA .

Reading sequences were compared by calculating the Levenshtein distance between two reading sequences of text nodes. The Levenshtein distance is calculated by counting the minimal number of edit operations such as insertions, deletions or substitutions needed to transform one string into the other. For example, when the Levenshtein distance between two reading sequences, where visited nodes are represented as a string of letters $\mathrm{ABCD}$ and 
AEFDA, is calculated, two substitutions and one insertion are needed to transform ABCD into AEFDA. The letter B is substituted with $\mathrm{E}$, the letter $\mathrm{C}$ is substituted with $\mathrm{F}$, and at the end of the string the letter A has to be added, resulting in a Levenshtein distance of 3. As the Levenshtein distance is a count of the smallest number of operations needed to transform one string into the other, lower values mean higher similarity between strings.

The degree of coherence of the reading sequences was computed as the sequence of text nodes visited the first time compared with a model sequence of text nodes of high coherence. The model sequence followed a top to bottom reading sequence within each branch and a left to right reading sequence between branches of the hierarchy found by Amadieu et al. (2009) when studying navigation behaviour with hierarchical overviews. Thus, the model sequence followed the path 'Project management', 'Project initiation', 'Planning procedure', 'Data gathering', 'Data analysis', etc. (see Figure 1.). For the purposes of analysis, the model node sequence was denoted as a string of the first 15 letters of the English alphabet, ABCDEFGHIJKLMNO, where A stood for 'Project management', B for 'Project initiation' and so forth. If, for example, a participant followed the path ABCDEFGHIJKLMN then the Levenshtein between the model sequence and the actual navigation sequence followed by the participant was 1 . In this case, the 14 nodes visited followed the same sequence as the model, but the last node denoted with the letter ' $O$ ' was not visited at all, requiring an edit operation, namely an insertion. In addition, the homogeneity of navigation paths per condition was calculated, with repeated visits to text nodes also included. Homogeneity was the measure of the degree of similarity between the navigation paths of participants. Homogeneity was included, as previous research about multiple electronic document comprehension indicated that a condition with lower homogeneity can lead to higher comprehension than a condition with higher homogeneity, when the level of cohesion of the navigation paths (i.e., degree of overlap in arguments between subsequently read texts ) 
between conditions is similar (Salmerón, Gil, Bråten, \& Strøms $\varnothing, 2010$ ). For this measure, the average Levenshtein distance for each participant as compared to every other participant in the same condition was computed, following Salmerón et al. Thus, for a participant in a condition with $\mathrm{X}$ participants, Levenshtein distance was calculated X-1 number of times, by comparing the navigation sequence of the participant with the navigation sequence of every other participant in the same condition. The Levenshtein distance values were then averaged to arrive at the homogeneity value used in further analysis.

\section{Results}

A criterion of at least two-thirds of the text nodes read (i.e., 10 text nodes or more) was established prior to statistical analysis for inclusion in the analyses. In line with this criterion, six participants were excluded from further analysis: three who did not visit any text nodes, one who visited one text node, and one who visited eight text nodes. One additional participant was excluded from the analysis because of scoring much lower on the post-test than on the prior-knowledge test ( $\Delta=-4$ points). The exclusion of this participant as an outlier is justified as all participants who visited at least ten text nodes scored at least as high or higher on the post-test than on the prior-knowledge test. The summary of the means and standard deviations of dependent variables in presented in Table2.

$$
\text { ***Insert Table } 2 \text { about here*** }
$$

\subsection{Prior Knowledge}


There were no significant differences between groups for prior knowledge $F(3,69)=0.16, M S E=2.71, p=.93$.

\subsection{Navigation}

As stated, 2X2 ANOVAs were conducted with the between subjects variables Type (dynamic, static) and Restriction (non-restricted, restricted) to ascertain whether there were differences between conditions for the following variables: time spent in the learning phase (Time), number of times the graphical overview was opened (NrOverview), number of times text nodes were opened (NrTextNodes) respectively, degree of coherence of the node sequence (Coherence) and homogeneity of the node sequence (Homogeneity). To normalize data, the square root transformed data of the variables NrOverview, NrTextNodes, Homogeneity and Coherence were entered into the analysis.

\subsubsection{Time.}

No significant differences were found between groups for time spent in the learning phase $F(3,69)=0.40, M S E=226087.93, p=.75$.

\subsubsection{Number of times the graphical overview was opened.}

Significant main effects were found for Type $F(1,69)=104.17, M S E=0.88$, $p=.0001, \eta p^{2}=.602$ and Restriction $F(1,69)=7.14, M S E=0.88, p=.009 \eta p^{2}=.094$ on NrOverview. In the dynamic conditions, the graphical overview was opened more frequently $(M=5.65 ; S D=1.08)$ than in the static conditions $(M=3.41 ; S D=0.95)$ and in the nonrestricted condition, the graphical overview was opened less frequently $(M=4.24, S D=0.16)$ than in the restricted conditions $(M=4.82, S D=0.16)$. There was also a significant 
interaction effect between Type and Restriction on NrOverview $F(1,69)=7.63, M S E=0.88$, $p=.007, \eta p^{2}=.100$ (Figure 4). Simple effects analysis revealed that in the dynamic nonrestricted condition, the graphical overview was opened more often than in the static nonrestricted condition $F(1,70)=26.23, M S E=0.96, p=.0001, \eta p^{2}=.273$ and that in the dynamic restricted condition the graphical overview was opened more often than in the static restricted condition $F(1,70)=75.94, M S E=0.96, p=.0001, \eta p^{2}=.520$. Furthermore, the graphical overview in the dynamic restricted condition was opened more often than in the static non-restricted condition $F(1,70)=5.59, M S E=2.17, p=.02, \eta p^{2}=.074$ but there was no significant difference between the static, non-restrictive and static, restrictive conditions $F(1,70)=0.02, M S E=2.17, p=.88$.

*** Insert Figure 4 about here $* * *$

\subsubsection{Number of times text nodes were opened.}

Analysis revealed significant main effects of Type $F(1,69)=23.05, M S E=1.25$, $p=.0001, \eta p^{2}=.250$ and Restriction $F(1,69)=20.05, M S E=1.25, p=.0001, \eta p^{2}=.225$ on NrTextNodes. Text nodes were opened more often in the static $(M=6.84, S D=0.18)$ than in the dynamic conditions $(M=5.58, S D=0.19)$ and more often in the restricted $(M=6.79$, $S D=0.19)$ than in the non-restricted conditions $(\mathrm{M}=5.62, \mathrm{SD}=0.19)$. There was no significant interaction effect between Type and Restriction on the variable NrTextNodes $F(1,69)=0.01, M S E=1.25, p=0.91$.

\subsubsection{Coherence.}

Significant main effects of Type $F(1,69)=7.85, M S E=1.07, p=.009 \eta p^{2}=.096$ and Restriction $F(1,69)=17.32, M S E=1.07, p=.0001, \eta p^{2}=.190$ were found for the 
Coherence. The reading sequence was more coherent in the dynamic conditions $(M=1.45$, $S D=0.95)$ than in the static $(M=2.10, S D=1.29)$. Coherence was also greater in the restricted conditions $(M=1.31, S D=1.14)$ than in the non-restricted $(M=2.30, S D=1.03)$. There was no significant interaction $F(1,69)=1.46, M S E=1.07, p=.231$.

\subsubsection{Homogeneity.}

Significant main effects of Type $F(1,69)=33.82, M S E=0.56, p=.0001 \eta p^{2}=.329$ and Restriction $F(1,69)=37.52, M S E=0.56, p=.0001 \eta p^{2}=.352$ were also found for Homogeneity. The nodes sequences showed more homogeneity in the dynamic conditions $(M$ $=5.67, S D=0.96)$ than in the static conditions $(M=6.71, S D=0.88)$ and were more homogenous in the non-restricted conditions $(M=5.67, S D=0.81)$ than in the restricted conditions $(M=6.76, S D=0.99)$. The interaction between factors was not significant $F(1,69)=0.59, M S E=0.56, p=.445$.

\subsection{Mental effort during the learning phase}

Scores for mental effort during the learning phase were analysed as within subjects for the variable Time-on-task, using repeated measures ANOVA for the first five points of time measurement. At t4, all participants were still in the learning phase. Five participants exited the learning phase before 5 and their missing values were substituted by their group means for this time point. The percentage of missing values at t5 was $6.85 \%$. At t6, eighteen participants were no longer in the learning phase, therefore time points later than t5 were excluded from the analysis.

The analysis yielded a significant main effect of Time-on-task with the GreenhouseGeisser correction $F(2.95,203.83)=3.79, M S E=1.29, p=.012, \eta p^{2}=.052$. The test of 
within-subjects contrasts showed that the levels of the variable Time-on-task described a linear relationship $F(1,69)=6.28, p=.015, \eta p^{2}=.083$. Bonferroni adjustment of multiple comparisons showed a significant difference between time points t4 and t5 ( $p=.019)$ and between $\mathrm{t} 1$ and $\mathrm{t} 5(p=.028)$ The linear trend seemed to be mostly due to rising mental effort scores in the dynamic restricted and static non-restricted conditions at $t 5$, while in the dynamic non-restricted and static restricted condition this rise was much less pronounced (Figure 5).

*** Insert Figure 5 about here $* * *$

\subsection{Post-test Measurements for Comprehension and Mental Effort}

Post-test knowledge scores and mental effort scores related to test-items during the test phase were analysed with separate $2 \mathrm{X} 2$ ANOVAs.

\subsubsection{Comprehension scores for multiple-choice test 1.}

No main effects of Type $F(1,69)=0.18, M S E=1.67, p=.68$ or Restrictions $F(1,69)=0.41, M S E=1.67, p=.52$ were found for comprehension, however the interaction between these factors was significant $F(1,69)=10.47, M S E=1.67, p=.002, \eta p^{2}=.132$ (Figure 6). Simple effects analysis showed that scores in the dynamic non-restricted condition were significantly higher $F(1,70)=3.86, M S E=1.66, p=.053, \eta p^{2}=.052$ than in the static non-restricted condition and that scores in the dynamic restricted condition were significantly lower than in the static restricted condition $F(1,70)=6.65, M S E=1.66, p=.012, \eta p^{2}=.087$. The scores in the dynamic non-restricted condition were significantly higher than in the dynamic restricted condition $F(1,70)=7.12, M S E=1.65, p=.009, \eta p^{2}=.092$. The difference between the static non-restricted condition and static restricted condition was 
marginally significant $F(1,70)=3.68, M S E=1.65, p=.059, \eta p^{2}=.050$ with higher scores in the static restricted condition.

$* * *$ Insert Figure 6 about here $* * *$

\subsubsection{Mental effort scores for multiple-choice test 1 .}

There were no significant differences between conditions for mean mental effort $F(3,69)=1.59, M S E=1.44, p=.2$.

\subsubsection{Comprehension scores for multiple-choice test 2.}

There were no significant main or interaction effects found for knowledge scores for multiple-choice test $2 F(1,69)=0.731, M S E=2.19, p=.54$. To account for the different pattern found for multiple-choice test 1 and multiple-choice test 2, additional analyses were conducted. Paired sample t-tests revealed that on average participants scored significantly higher on multiple-choice test 1 than on multiple-choice test $2 t(72)=2.45, p=.017, r^{2}=.077$ $(M=8.60, S D=1.36$ vs. $M=8.16, S D=1.47)$, spent significantly more time answering multiple-choice test 1 than multiple-choice test $2 t(72)=8.03, p=.0001, r^{2}=.472$ $(M=353.78, S D=128.60$ vs. $M=254.71, S D=68.85)$ and exerted more mental effort during multiple-choice test 1 than during multiple-choice test $2 t(72)=3.03, p=.003$, $r^{2}=.112(M=3.45, S D=1.21$ vs. $M=3.17, S D=1.22)$.

\subsubsection{Mental effort scores for multiple-choice test 2.}

There were no significant differences between conditions for mean mental effort $F(3,69)=1.77, M S E=1.15, p=.161$. 


\subsubsection{Comparison of multiple choice tests 1 and 2.}

The two multiple-choice tests used in the post-test phase yielded inconsistent results. Whereas test 1 showed a significant interaction effect, test 2 showed no significant differences. As test 1 and test 2 consisted of related items, the test items of test 2 might have come across as overly familiar. Participants spent significantly more time on test 1 than on test 2 , yet they scored significantly lower an exerted less mental effort on test 2 than test 1 , which suggests that participant might have felt less motivated and therefore paid less attention to test 2 . It is therefore probably safe to assume that the results of test 2 reflected not only the influence of the experimental manipulation, but also of test 1 , which makes the interpretation the outcomes of test 2 problematic. Therefore, test 2 , and the associated mental effort scores are not considered in the discussion.

\section{Discussion}

The hypotheses predicting that conditions with a similar degree of the coherence of the node sequence need not lead to similar levels of learning outcomes when the degree of redundant use differs, appears to be largely confirmed. If coherence of the reading sequence of text nodes had a decisive influence, the dynamic non-restricted en static restricted conditions with the highest levels of comprehension should both have showed a greater degree of coherence than the other two conditions. This was, not the case, as no significant interaction for coherence was found. What was found instead was that both dynamic overviews lead to a more coherent reading sequence than the two static graphical overviews, and that the restricted overviews led to higher coherence than the non-restricted overviews.

A noteworthy finding is that the dynamic non-restricted overview led to lower coherence than both the dynamic restricted and static non-restricted overviews. This is 
contrary to the expected highest coherence in the dynamic non-restricted condition, indicating that the restricted navigation imposed here (i.e., allowing access to just the child and parent nodes) results in a closer adherence to a top to bottom, left to right reading sequence with hierarchical overviews than when learners are free decide on their reading sequence. However, the higher coherence of the restricted conditions was not associated with a higher comprehension than the dynamic non-restricted condition, in fact the dynamic restricted condition scored significantly lower. This finding offers further support to the hypotheses suggesting that the degree of coherence can only partly account for learning outcomes. Just as conditions with comparable levels of coherence, conditions with similar homogeneity did not lead to a similar level of comprehension. Here too no interaction was found, with dynamic overviews leading to more homogeneity in reading sequence than static overviews, but the restricted conditions led to a less homogeneous reading sequence than the two non-restricted conditions. Thus, a condition associated with high coherence and comprehension, such as the static non-restricted condition in the present study, can be at the same time low on homogeneity, which is line with the findings of Salmerón at all. (2010).

Closer examination of navigation data concerning the number of times text nodes were opened sheds some light on the origin of the less homogeneous reading sequence in the restricted conditions. When participants in restricted conditions diverged from each other, it lead them down different navigation paths as a consequence of the restrictions imposed at the follow-up steps. Turning back involved retracing the path taken in the opposite direction, resulting in higher number of visits to individual text-nodes in restricted conditions than in non-restricted conditions where direct jumps between distant nodes were allowed. It could be argued that just as in the case of graphical overviews, repeated involuntary and redundant visits to individual text nodes might also cause extraneous load. The results, however, do not support this explanation, as the two restricted conditions did not differ significantly for the 
frequency that text nodes were opened, but did differ for comprehension, with the dynamic restricted condition leading to the lowest level of comprehension of all the conditions. The finding that the dynamic restricted overview was opened more frequently than the static restricted overview may indicate that the higher level of use of the dynamic restricted overview was indeed redundant from the point of following a coherent nodes sequence, as the static restricted condition led to just as high a coherence.

Although differences between conditions did not reach significance, the mental effort scores during the latter stages of the learning phase followed the expected pattern; the higher the mental effort in a condition, the lower the level of comprehension. Moreover, mental effort across conditions was significantly higher at the latter stages of the learning phase, adding support to Salmerón et al's (2009) finding that graphical overviews may be less helpful at the end than at the beginning of the learning phase. In addition, mental effort exerted during the test-phase was measured. However, mental effort related to post-test showed a slightly different picture for the static non-restricted condition than in the learning phase: in the learning phase it lead to the second highest mental effort, whereas in the test phase to the lowest mental effort of all the conditions. As participants in this conditions scored low on comprehension, low mental effort scores may be an indication for loss of motivation (Paas, Tuovinen, Van Merriënboer, \& Darabi, 2005). The overview in this condition was not a navigation device as in the other three conditions which not only negatively comprehension, but might have been also experienced as not very useful by the participants, with a negative effect on motivation.

\subsection{Limitations}


The differences between conditions, both during the learning- and test phase, were not significant which poses the question whether self-reported mental effort is a sensitive enough measure to capture actual differences in mental effort related to differences in experimental conditions (De Jong, 2009). A study conducted by Antonenko and Niederhouser (2010) indicated that tracking the subtle changes in extraneous load might indeed require a more sensitive measure than self-reported mental effort and should be collected during the learning phase. Examining the effects of leads in pop-up windows offering a short explanation of hyperlinks, the authors compared cognitive load scores obtained by EEG-based measures of brain activity during the learning phase with self-report scores of mental effort gathered following the learning task. They hypothesised that leads might decrease split-attention caused by disruptions in comprehension when navigating between nodes. The EEG-measures showed significantly lower brain activation in the leads-augmented hypertext, but the selfreport measure of mental effort following the learning phase failed to show any difference. Furthermore, the pattern found in the present study also suggests that extraneous load may undergo significant changes over time which would be difficult to capture in a single score of mental effort following the learning phase.

The static non-restricted condition where each text node had hyperlinks leading to all the other text nodes did not induce the highest mental effort as expected. It was hypothesised that extraneous load will be the highest in this condition due to very low coherence. A possible explanation for this finding might be that the hypertext used in the present study had too few text-nodes to have a marked negative impact on coherence. A higher number of hyperlinks leading to text nodes were earlier shown to lead to a less coherent reading sequence than a lower number of hyperlinks in a hypertext with more text nodes (Madrid, Van Oostendorp, \& Puerta Melguizo, 2009). The finding that text nodes were read in a more or less equally coherent reading sequence with the static non-restricted overview and with the 
dynamic non-restricted overview, suggests that coherence was never so low as to in itself have a major impact on extraneous load, even without an overview relevant for navigation coherence. The low number of text nodes may also explain why the dynamic non-restricted and static restricted conditions both lead to a similar level of comprehension, instead of the hypothesised lower comprehension for the dynamic non-restricted condition. With more text nodes, the difference in redundant use between these two conditions might have been larger than in the present set-up, exerting a more differentiated influence on both mental effort and comprehension. Results concerning the frequency with which the graphical overview was opened in the dynamic conditions supports this explanation; although the dynamic restricted overview was opened more frequently than the dynamic non-restricted overview, this difference was not significant. The high scores on post-test comprehension scores in these conditions also offer an alternative explanation, suggesting a possible ceiling effect.

A further limitation concerns how mental effort scores were collected during the learning phase. The question about mental effort appeared in a pop-up window covering the screen which may have acted as an interference by disrupting the reading process. However, the interference caused by this method of mental effort assessment was present in all conditions and therefore probably had no marked influence on the main outcomes of this study. Ideally methods which are less intrusive (e.g., EEG measures) should be used to assess mental effort in the course of learning. However, in the present study the use of such methods were not practically feasible.

\subsection{Conclusion}

The effects of graphical overviews in hypertext learning environments seem to be multifold and, therefore, should be studied along multiple dimensions. The need for a 
multidimensional approach for studying hypertext comprehension was previously identified by Amadieu et al. (2010). The present study applied multidimensionality by focusing on the influence of the coherence of the node sequence in conjunction with the extraneous load induced by graphical overviews on comprehension. Results indicated that the coherence of node sequence induced by graphical overviews cannot account for learning outcomes in isolation. Instead, the use of, especially dynamic overviews, for enhancing coherence at latter stages of the learning process may become redundant, having a negative influence on comprehension. However, the lack of significant differences for mental effort between conditions associated with differing levels of graphical overview use makes clear the need for follow-up research in this area. Future research employing more sensitive and continuous measures of cognitive load during the learning phase might offer a more in-depth view of the complex relationship which seems to exist between graphical overviews and the degree of coherence they induce in hypertext learning. 


\section{References}

Amadieu, F., Tricot, A., \& Mariné, C. (2010). Interaction between prior knowledge and concept-map structure on hypertext comprehension, coherence of reading orders and disorientation. Interacting with Computers, 22(2), 88-97

Amadieu, F., Van Gog, T., Paas, F., Tricot, A., \& Mariné, C. (2009). Effects of prior knowledge and concept-map structure on disorientation, cognitive load, and learning. Learning and Instruction, 19, 376-386.

Antonenko, P. D., \& Niederhauser, D. S. (2010). The influence of leads on cognitive load and learning in a hypertext environment. Computers in Human Behavior, 26(2), 140-150.

Chalmers, P. A. (2003). The role of cognitive theory in human-computer interface. Computers in Human Behavior, 19(5), 593-607.

Chandler, P., \& Sweller, J. (1991). Cognitive load theory and the format of instruction. Cognition and Instruction, 8, 292-332.

Chandler, P., \& Sweller, J. (1992). The split-attention effect as a factor in the design of instruction. British Journal of Educational Psychology, 62, 233-246.

Chandler, P., \& Sweller, J. (1996). Cognitive load while learning to use a computer programme. Applied Cognitive Psychology, 10, 151-170.

Chen, S.Y., Fan, J. P., \& Macredie, R. D. (2006). Navigation in hypermedia learning systems: Experts vs. novices. Computers in Human Behavior, 22, 251-266.

Conklin, J. (1987). Hypertext: An introduction and survey. IEEE Computer, 20, 17-41.

De Jong, T. (2009). Cognitive load theory, educational research, and instructional design: Some food for thought. Instructional Science, 38(2), 105-134.

DeStefano, D., \& LeFevre, J. (2007). Cognitive load in hypertext reading: A review. Computers in Human Behavior, 23, 1616-1641. 
Fayol, M., \& Rouet, J.-F. (2008). Memory processes in text and multimedia comprehension: Some reflections and perspectives. In J.F. Rouet, R. Lowe, \& W. Schnotz (Eds.), Understanding Multimedia Documents (pp. 267-280). Boston, MA: Springer.

Hannafin, M. J., \& Land, S. M (1997). The foundations of and assumptions of technologyenhanced student centered learning environments. Instructional Science, 25, 167-202.

Kalyuga, S., Ayres, P., Chandler, P., \& Sweller, J. (2003). The expertise reversal effect. Educational Psychologist, 38, 23-31.

Kalyuga, S., Chandler, P., \& and Sweller, J. (2000). Incorporating learner experience into the design of multimedia instruction. Journal of Educational Psychology, 92, 126-136.

Kintsch, W. (1988). The role of knowledge in discourse comprehension: A constructionintegration model. Psychological Review, 95, 163-182.

McDonald, S., \& Stevenson, R. J. (1996). Disorientation in hypertext: The effects of three text. structures on navigation performance. Applied Ergonomics, 27(1), 61-68.

McDonald, S., \& Stevenson, R. J. (1998). Navigation in hyperspace: An evaluation of the effects of navigational tools and subject expertise on browsing and information retrieval in hypertext. Interacting with Computers, 10, 129-142.

McNamara, D. S.,\& Kintsch,W. (1996). Learning from text: Effect of prior knowledge and text coherence. Discourse Processes, 22, 247-288.

McNamara, D. S., Kintsch, E., Butler Songer, N., \& Kintsch, W. (1996). Are good texts always better? Interactions of text coherence, background knowledge, and levels of understanding in learning from text. Cognition and Instruction, 14, 1-43.

Madrid, R. I., Van Oostendorp, H. \& Puerta Melguizo, M. C. (2009). The effects of the number of links and navigation support on cognitive load and learning with hypertext: The mediating role of reading order. Computers in Human Behavior, 25, 66-75. 
Madrid, R. I., \& Cañas, J. J. (2009). The effect of reading strategies and prior knowledge on cognitive load and learning with hypertext. The Ergonomics Open Journal, 2, 124-132.

Marchionini, G., \& Shneiderman, B. (1988). Finding facts vs. browsing knowledge in hypertext systems. IEEE Computer, 27(1), 70-80.

Mayer, R. E. (2005). Principles for managing essential processing in multimedia learning: Coherence, signalling, redundancy, spatial contiguity and temporal contiguity principles. In R. E. Mayer (Ed.), Cambridge handbook of multimedia learning (pp. 183- 200). New York: Cambridge University Press.

Müller-Kalthoff, T., \& Möller, J. (2003). The effects of graphical overviews, prior knowledge, and self-concept on hypertext disorientation and learning achievement. Journal of Educational Multimedia and Hypermedia, 12, 117-134.

Müller-Kalthoff, T., \& Möller, J. (2006). Browsing while reading: Effects of instructional design and learners prior knowledge. Research in Learning Technology 14(2), 183-198.

Paas, F. (1992). Training strategies for attaining transfer of problem-solving skill in statistics: A cognitive-load approach. Journal of Educational Psychology, 84, 429-434.

Paas, F., Tuovinen, J. E., Van Merriënboer, J. J. G., \& Darabi, A. A. (2005). A motivational perspective on the relation between mental effort and performance: Optimizing learner involvement in instruction. Educational Technology Research and Development 53(3), 2534.

Patel, S. C., Drury, C. G., \& Shalin, V. L. (1998). Effectiveness of expert semantic knowledge as a navigational aid within hypertext. Behaviour and Information Technology, 17, 313324.

Potelle, H., \& Rouet, J.-F. (2003). Effects of content representation and reader's prior knowledge on the comprehension of hypertext. International Journal of Human-Computer Studies, 58, 327-345. 
Puerta Melguizo, M. C., Salmerón, L., \& Madrid, R. I., \& Van Oostendorp, H. (2009). The role of reading order and monitoring skills during hypertext comprehension. The Ergonomics Open Journal, 2, 97-103.

Reader, W., \& Hammond, N. (1994). Computer-based tools to support learning from hypertext: Concept mapping tools and beyond. Computers \& Education 22, 99-106.

Salmerón, L., Baccino, T., Cañas, J. J., Madrid, R. I., \& Fajardo, I. (2009). Do graphical overviews facilitate or hinder comprehension in hypertext? Computers \& Education, 53(4), 1308-1319.

Salmerón, L., Cañas, J. J., Kintsch, W., \& Fajardo, I. (2005). Reading strategies and hypertext comprehension. Discourse Processes, 40, 171-191.

Salmerón, L., Gil, L., Bråten, I., \& Strømsø, H. (2010). Comprehension effects of signalling relationships between documents in search engines. Computers in Human Behavior, 26, 419-426.

Salomon, G., \& Almog. T. (1998). Educational psychology and technology: A matter of reciprocal relations. Teachers College Record, 100(1), 222-241.

Schnotz, W., \& Heiß, A. (2009).Semantic scaffolds in hypermedia learning environments. Computers in Human Behavior, 25, 371-380. Shapiro, A., \& Niederhauser, D. (2004).

Learning from hypertext: Research issues and findings. In. D. H. Jonassen, (Ed.), Handbook of research on educational communications and technology $\left(2^{\text {nd }}\right.$ ed.), (pp. 605620). Mahwah, NJ: Lawrence Erlbaum Associates.

Shin, E., Schallert, D., \& Savenye, C. (1994). Effects of learner control, advisement, and prior knowledge on young students' learning in hypertext environments. Educational Technology Research and Development, 42, 33-46.

Sweller, J., Van Merriënboer, J. J. G., \& Paas, F. (1998). Cognitive Architecture and Instructional Design. Educational Psychology Review, 10, 251-296. 
Van Gog, T., \& Paas, F. (2008). Instructional efficiency: Revisiting the original construct in educational research. Educational Psychologist, 43(1), 16-26.

Van Merriënboer, J. J. G., \& Kirschner, P. A. (2012). Ten steps to complex learning (Second edition). New York: Taylor \& Francis. 


\section{Table 1}

Expectancy table for the outcome variables coherence, learning outcomes, mental effort during the learning phase, and the frequency with which the overview is opened.

\begin{tabular}{cccccc}
\hline & \multicolumn{2}{c}{ Dynamic } & & \multicolumn{2}{c}{ Static } \\
\cline { 2 - 3 } \cline { 5 - 6 } Conditions & restricted & non-restricted & & restricted & non-restricted \\
\hline Coherence & Medium & High & & Medium & Low \\
Learning outcomes & Low & Medium & & High & Low \\
Mental effort & High & Medium & & Low & High \\
Freq. Overview Use & High & Medium & & Low & Low \\
\hline
\end{tabular}




\section{Table 2}

Means and standard deviations for the number of times the graphical overview was opened, number of times text nodes were opened, coherence of the node sequence, homogeneity of the nodes sequence and comprehension and mental effort for post-test 1 and post-test 2.

\begin{tabular}{cccccc}
\hline & \multicolumn{2}{c}{ Dynamic } & & \multicolumn{2}{c}{ Static } \\
\cline { 6 - 6 } \cline { 5 - 6 } Conditions & Restricted & Non-restricted & & Restricted & Non-Restricted \\
Freq. Overview & $6.25(1.08)$ & $5.05(0.69)$ & & $3.40(0.89)$ & $3.42(1.03)$ \\
Freq. Text nodes & $6.18(1.09)$ & $4.97(0.70)$ & & $7.41(1.39)$ & $6.26(1.12)$ \\
Coherence & $1.11(0.90)$ & $1.80(0.91)$ & & $1.50(1.30)$ & $2.74(0.93)$ \\
Homogeneity & $6.28(0.92)$ & $5.06(0.53)$ & & $7.17(0.87)$ & $6.22(0.61)$ \\
Post test 1 & $7.94(1.60)$ & $9.12(0.99)$ & & $9.05(1.36)$ & $8.26(1.45)$ \\
Effort post test 1 & $3.99(1.31)$ & $3.31(1.23)$ & & $3.36(1.17)$ & $3.19(1.11)$ \\
Post test 2 & $8.06(1.71)$ & $7.76(1.89)$ & & $8.40(1.14)$ & $8.37(1.12)$ \\
Effort post test 2 & $3.75(1.46)$ & $2.96(1.22)$ & & $2.96(0.78)$ & $3.06(1.28)$ \\
\hline
\end{tabular}




\section{Figure Captions}

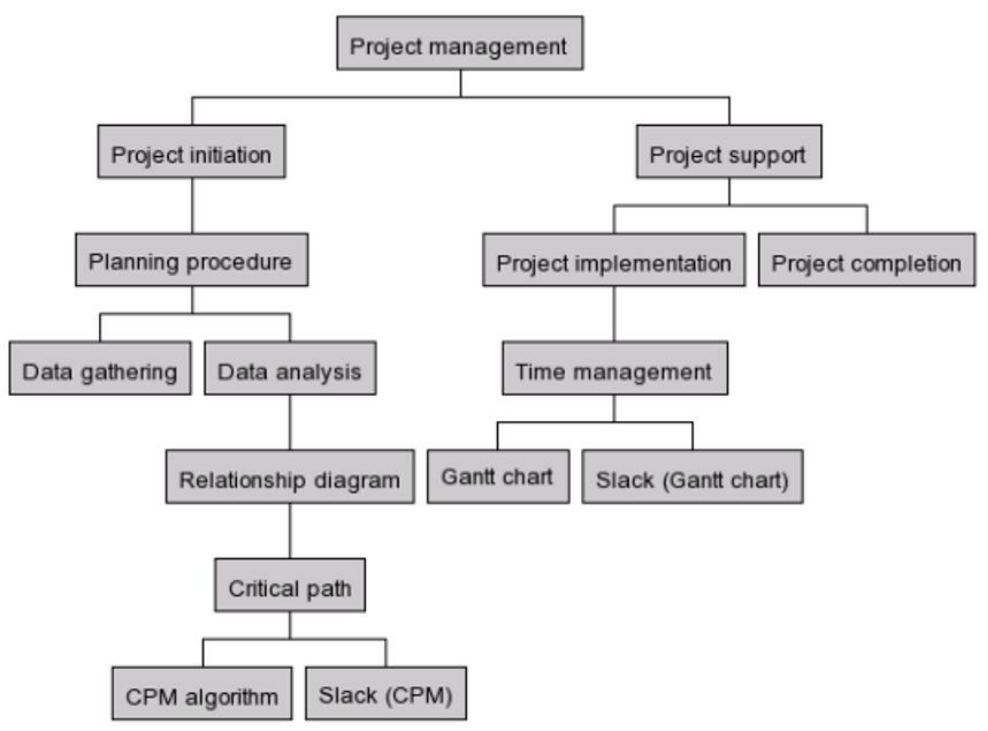

Figure 1. The hierarchical structure presented in the graphical overviews.

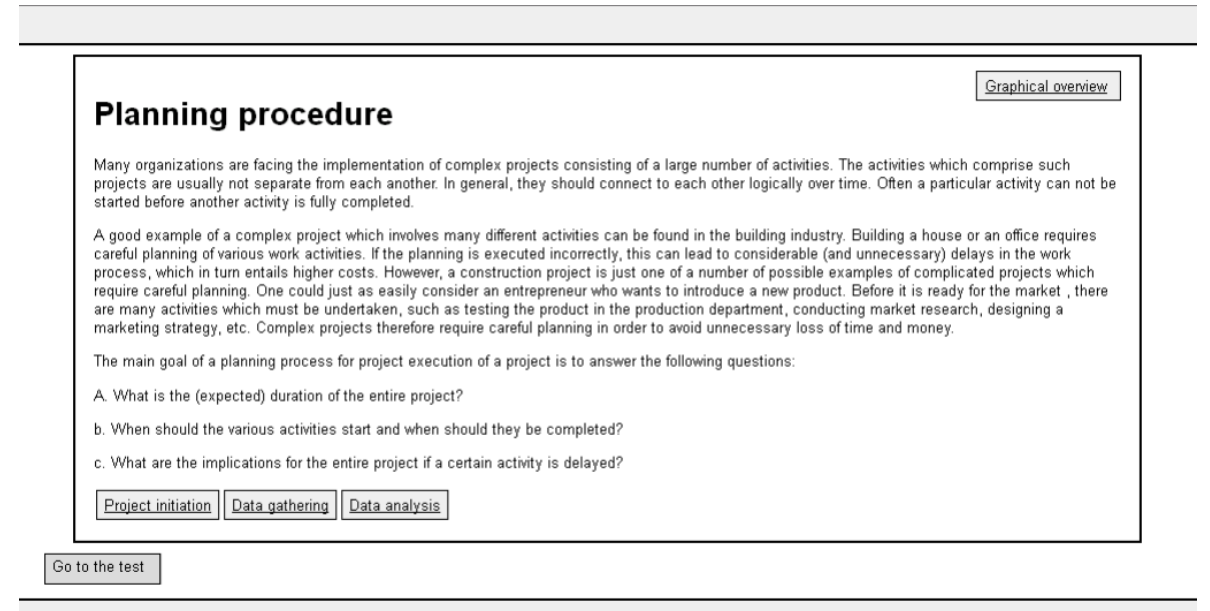

Figure 2. Example of a text node in the static restricted condition. 


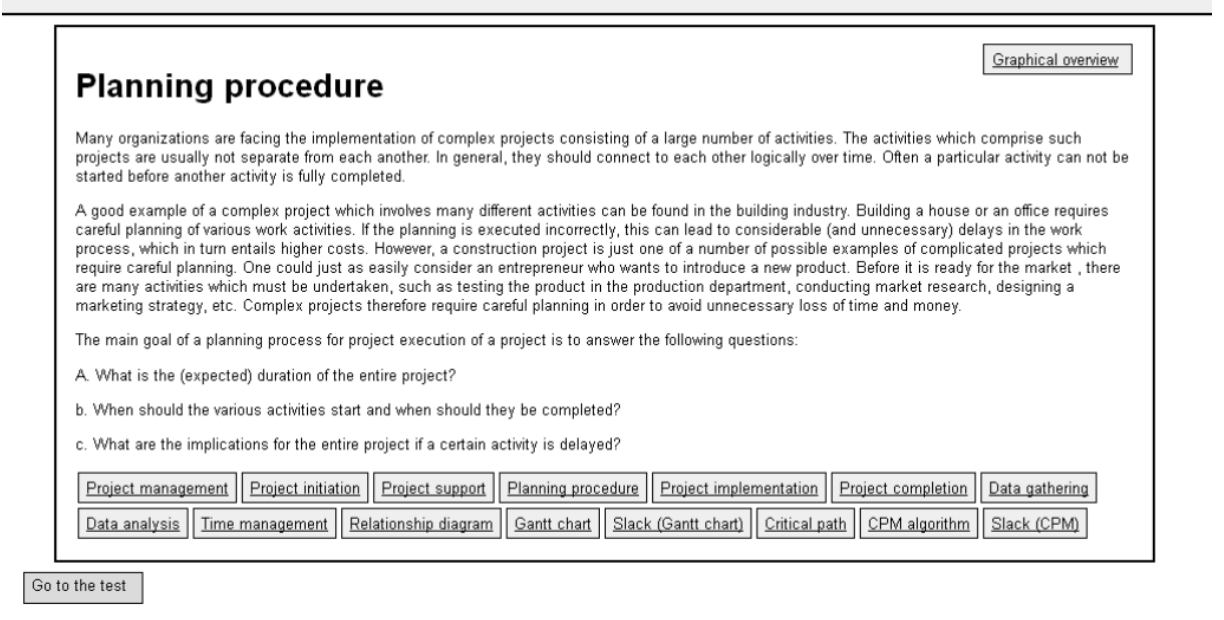

Figure 3. Example of a text node in the static non-restricted condition.

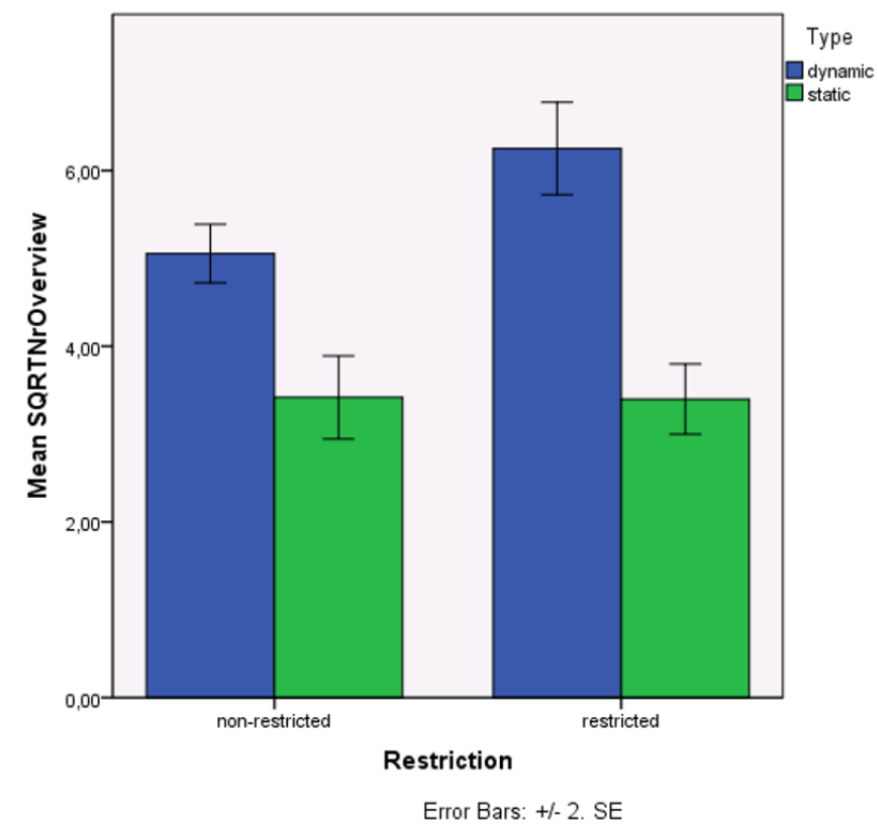

Figure 4. The square root of the number of times the graphic overview were visited. Error bars represent standard errors. 


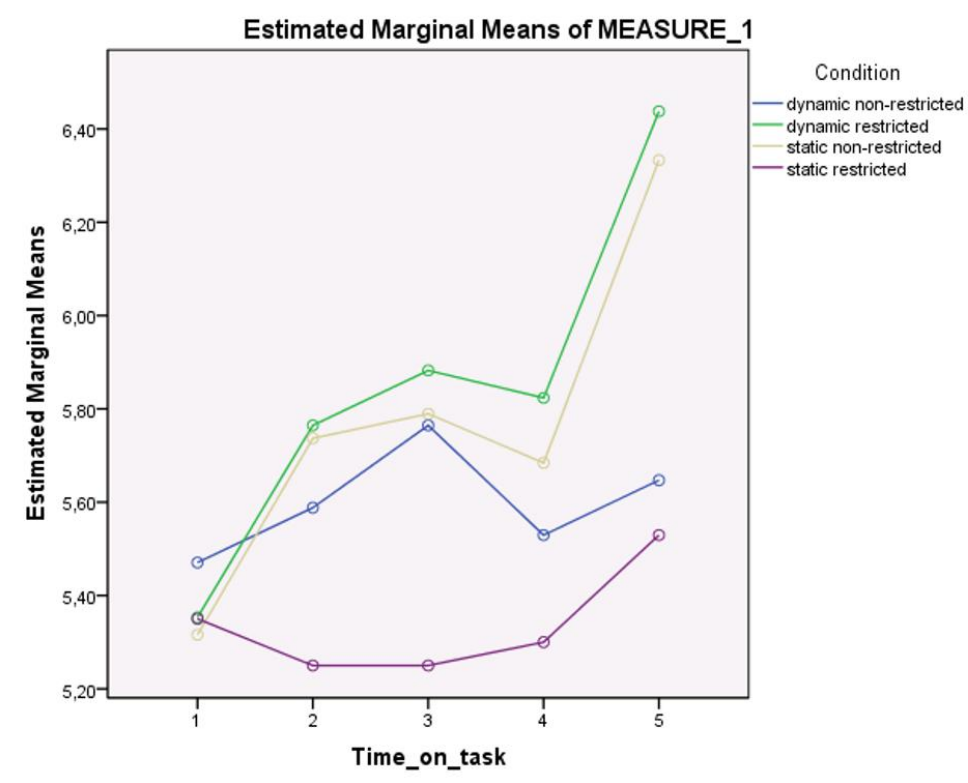

Figure 5. Mental effort scores during the first 25 minutes of the learning phase.

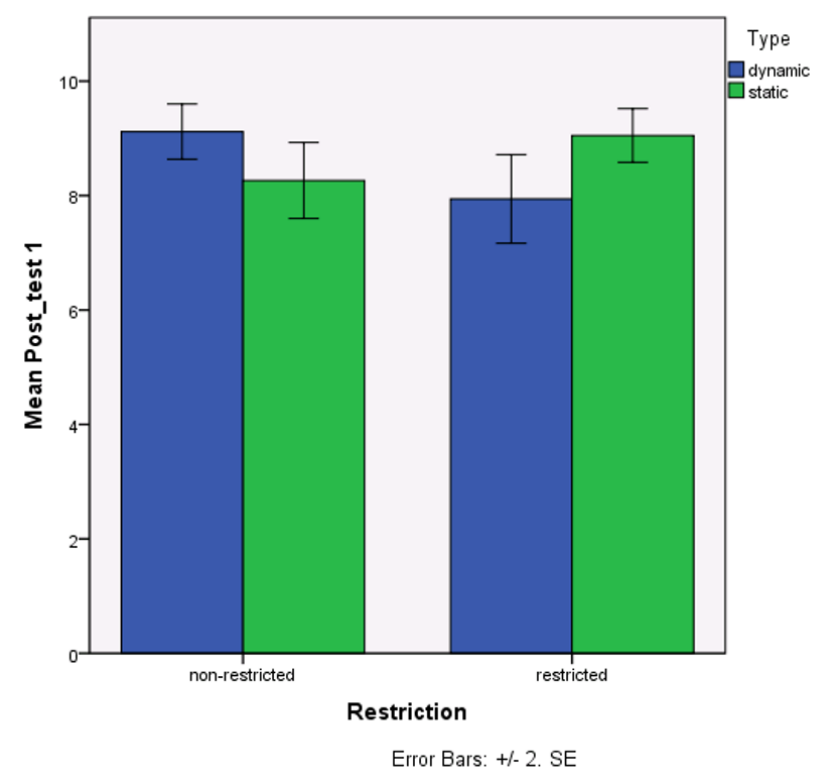

Figure 6. Post-test comprehension scores for multiple-choice test 1 of ten items. Error bars represent standard errors. 